\title{
Fast on-wafer electrical, mechanical, and electromechanical characterization of piezoresistive cantilever force sensors
}

\author{
G. Tosolini, ${ }^{1}$ L. G. Villanueva ${ }^{2}$ F. Perez-Murano, ${ }^{1}$ and J. Bausells ${ }^{1, a)}$ \\ ${ }^{1}$ Instituto de Microelectrónica de Barcelona IMB-CNM (CSIC), 08193 Bellaterra, Spain \\ ${ }^{2}$ California Institute of Technology, Pasadena, California 91125, USA
}

(Received 1 July 2011; accepted 6 December 2011; published online 11 January 2012)

\begin{abstract}
Validation of a technological process requires an intensive characterization of the performance of the resulting devices, circuits, or systems. The technology for the fabrication of micro and nanoelectromechanical systems (MEMS and NEMS) is evolving rapidly, with new kind of device concepts for applications like sensing or harvesting are being proposed and demonstrated. However, the characterization tools and methods for these new devices are still not fully developed. Here, we present an on-wafer, highly precise, and rapid characterization method to measure the mechanical, electrical, and electromechanical properties of piezoresistive cantilevers. The setup is based on a combination of probe-card and atomic force microscopy technology, it allows accessing many devices across a wafer and it can be applied to a broad range of MEMS and NEMS. Using this setup we have characterized the performance of multiple submicron thick piezoresistive cantilever force sensors. For the best design we have obtained a force sensitivity $\Re_{F}=158 \mu \mathrm{V} / n \mathrm{~N}$, a noise of $5.8 \mu \mathrm{V}(1 \mathrm{~Hz}-1$ $\mathrm{kHz}$ ) and a minimum detectable force of $37 p \mathrm{~N}$ with a relative standard deviation of $\sigma_{r} \approx 8 \%$. This small value of $\sigma_{r}$, together with a high fabrication yield $>95 \%$, validates our fabrication technology. These devices are intended to be used as bio-molecular detectors for the measurement of intermolecular forces between ligand and receptor molecule pairs. (C) 2012 American Institute of Physics. [doi:10.1063/1.3673603]
\end{abstract}

\section{INTRODUCTION}

Microfabricated cantilevers have been used to study numerous physical, chemical, and biological phenomena. ${ }^{1}$ Efficient, low-noise transduction of the cantilever motion is critical to the best sensor performance. To that end, optical detection has been commonly used over the past three decades; typically the setup is based on the reflection of a laser beam focused on the cantilever surface. Although optical methods present high displacement resolution and are extensively used, they lose efficiency when downscaling the cantilever dimensions. This limits the applicability of optical detection for cantilevers with high force sensitivity and resolution. ${ }^{2}$ One way to overcome this problem consists in implementing an electrical read-out of the cantilever motion via, for example, piezoresistive effect. ${ }^{3}$

Silicon cantilevers with piezoresistive transduction were first fabricated at Stanford University ${ }^{4}$ for atomic force microscopy applications. After this, many groups have developed systems and applications based on cantilevers with integrated piezoresistors for force or displacement sensing. These include the development of atomic force microscopy (AFM) probes ${ }^{5-8}$ probes for magnetometry, ${ }^{9}$ the high force sensitivity achievement with $<100 \mathrm{~nm}$ thin cantilevers, ${ }^{10}$ the characterization of low force electrical contacts ${ }^{11}$ and the study of the ultimate piezoresistive sensitivity at low temperatures. ${ }^{12}$

We have previously presented the fabrication of cantilevers made of polycrystalline silicon using a commercial CMOS process flow together with integrated read-out

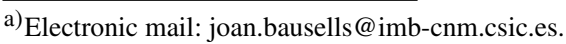

circuitry. ${ }^{13}$ However, polycrystalline silicon presents lower piezoresistive coefficients than crystalline silicon, which limits the sensitivity of the devices. Recently, we have fabricated crystalline silicon cantilevers, integrating n-doped piezoresistors $^{14}$ and n-MOSFET transistors ${ }^{15}$ using arsenic impurities in order to obtain shallower junctions. In order to maximize the devices sensitivity, the cantilevers are oriented along the non-standard $\langle 100\rangle$ direction on the wafer surface. ${ }^{16}$

A common denominator of different fabrication technologies is the need of testing large number of devices across several wafers. This need is not specific to MEMS and wafer scale testing has been used over the years to test electronic devices. What is specific to MEMS in general, and to piezoresistive cantilevers in particular, is the need to evaluate their sensitivity. That can be done by performing an accurate deflection of the cantilever while recording the resistance variation (i.e., the voltage variation on a Wheatstone bridge). In order to deflect the cantilever with high accuracy, various approaches have been used, such as a needle mounted on a bimorph piezodisk, ${ }^{17}$ a calibrated piezotube, ${ }^{18}$ an AFM in contact mode ${ }^{12}$ or in tapping mode, ${ }^{19}$ etc. A parallel approach, only valid for very low stiff devices, measures the resonant thermomechanically-driven displacement fluctuations. ${ }^{10,12}$ All these methods allowed a precise estimation of the device deflection sensitivity but were carried out on a chip level, therefore making technology validation a tedious and long process. An alternative which allows on-wafer testing is to deflect the cantilever by a needle mounted on a micromanipulator, ${ }^{8}$ but that is less accurate than the previously mentioned solutions. Here, we present a novel set of methods that allows on-wafer characterization with high 
accuracy, good repeatability, and unprecedented reduction in testing time. This set of methods, that can be adapted for a broad range of MEMS devices, is analyzed here for the particular case of piezoresistive cantilevers.

Section II presents the theoretical background to analyze the response of piezoresistive cantilevers. Section III describes the methods and experimental setup used to characterize the fabricated devices.

\section{THEORETICAL BACKGROUND}

The most important parameters determining the performance of a quasi-static force sensor based on piezoresistive cantilevers are the spring constant $k[\mathrm{~N} / \mathrm{m}]$, the force sensitivity $(\Delta \mathrm{V} / \mathrm{F})[\mathrm{V} / \mathrm{N}]$, the transducer noise $[\mathrm{V}]$ and the minimum detectable force (MDF) [N]. We use simple models which are adequate for our specific cantilevers. For the more general case other models are available which include issues such as non-uniform doping of the piezoresistors. ${ }^{20,21}$

\section{A. Spring constant: cantilever mechanical model}

The spring constant of a cantilever is defined as

$$
k=\frac{F}{\delta},
$$

and for a cantilever beam with constant cross section becomes

$$
k=3 \frac{E I}{L^{3}},
$$

where $F$ is the perpendicular force actuating at the cantilever end, $\delta$ its deflection, $L$ the cantilever length and $E I$ is the bending stiffness of the beam.

To calculate the spring constant of our multilayer, Ushaped cantilever (Figs. 1 and 2), we model it mechanically as follows. We divide it ideally into two parts: the first is the multilayer beam and the second is a single layer beam clamped to the first one both with constant cross sections. Thus, we obtain the cantilever spring constant,

$$
k=-\frac{3 E I_{1} E I_{2}}{\left(L_{1}-L_{2}\right)^{3}\left(E I_{1}-E I_{2}\right)-L_{2}^{3} E I_{2}},
$$

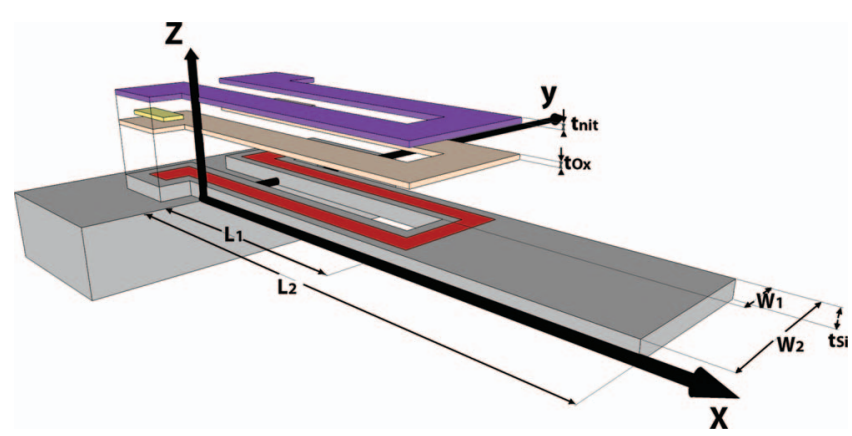

FIG. 1. (Color online) Exploded view of the piezoresistive cantilever. From the top there are: PECVD silicon nitride $\left(\mathrm{t}_{\mathrm{Nit}}=100 \mathrm{~nm}\right)$, thermal oxide $\left(\mathrm{t}_{\mathrm{Ox}}=38 \mathrm{~nm}\right)$, implanted crystalline silicon, and crystalline silicon $\left(\mathrm{t}_{\mathrm{Si}}=325 \mathrm{~nm}\right) . \mathrm{L}_{1}=125 \mu \mathrm{m}$ is half of the length of the piezoresistance and also the length of the multilayer part. $\mathrm{L}_{2}=250 \mu \mathrm{m}$ is the total length of the cantilever. The implanted piezoresistance is visible on the silicon. $z_{\mathrm{ti}}$ and $z_{\mathrm{di}}$ are the top and the bottom $\mathrm{z}$ coordinates of the layers, with $\mathrm{i}=1$ for silicon nitride, $\mathrm{i}=2$ for silicon oxide, and $\mathrm{i}=3$ for silicon.

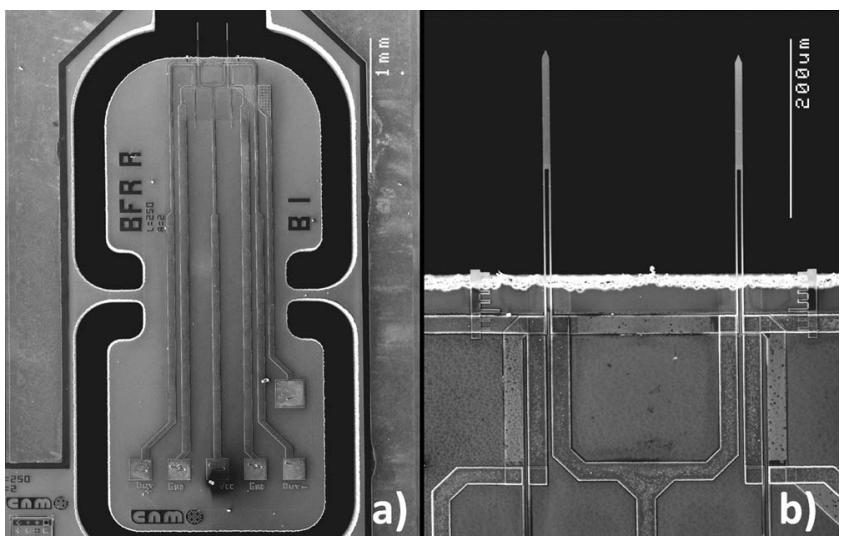

FIG. 2. (a) scanning electron microscope (SEM) image of a piezoresistive cantilever chip: two cantilevers are visible in the top part and the pads for contacting the chip are in the bottom part. (b) SEM image of two piezoresistive cantilevers. In total there are four piezoresistors per chip in a Wheatstone bridge configuration, two in the cantilevers and two in the substrate.

where $E I_{1}$ is the bending stiffness of the multilayer part and $E I_{2}$ is the bending stiffness of the second part, which are

$$
E I_{1}=\frac{1}{3} w_{1} \sum_{i=1}^{3} E_{i}\left(\left(z_{t i}-z_{0}\right)^{3}-\left(z_{d i}-z_{0}\right)^{3}\right),
$$

$$
E I_{2}=\frac{1}{12} w_{2} E_{3}\left(z_{t 3}-z_{d 3}\right)^{3},
$$

where the neutral axis is

$$
z_{0}=\frac{1}{2} \frac{\sum_{i=1}^{3} E_{i}\left(z_{t i}^{2}-z_{d i}^{2}\right)}{\sum_{i=1}^{3} E_{i}\left(z_{t i}-z_{d i}\right)},
$$

and $E_{1}, E_{2}$, and $E_{3}$ are the Young's moduli of silicon nitride, silicon oxide and crystalline silicon, respectively.

The complete description of the fabrication process can be found elsewhere. ${ }^{14}$

\section{B. Sensitivity: electromechanical model}

When a resistor is mechanically stressed, the relative variation of the resistance value $\left(\Delta \mathrm{R} / \mathrm{R}_{0}\right)$ is the result of dimensional resistor changes (length $l$, width $w$, and thickness $t)$ and change in resistivity $(\rho)$. In the case of low doped crystalline silicon, the change of the resistivity in certain directions (i.e. $\langle 100\rangle$ ) is larger than the dimensional changes by a factor of 50, thus the latter can be ignored. ${ }^{16}$ In case of a uniaxial stress $(\sigma)$ and parallel current flow, the relative resistance change is

$$
\frac{\Delta R}{R_{0}}=\frac{\Delta l}{l_{0}}-\frac{\Delta w}{w_{0}}-\frac{\Delta t}{t_{0}}+\frac{\Delta \rho}{\rho_{0}} \cong \frac{\Delta \rho}{\rho_{0}}=\pi_{l} \sigma,
$$

where the $\pi_{1}$ is the longitudinal piezoresistive coefficient for that certain direction. The cantilever under study is modeled as a linear elastic beam with a point load at the tip, as explained in Sec. II A. This leads to a longitudinal stress $\left(\sigma_{\mathrm{x}}\right)$ along the two legs of the cantilever that varies linearly along $\mathrm{x}$ and $\mathrm{z}$ and to a transverse stress along $\mathrm{y}$, that we consider negligible. Considering that the resistor, thus the current, and 
the stress are parallel and along the $\langle 100\rangle$ direction, we can write

$$
\frac{\Delta R}{R_{0}}=\pi_{11}\left\langle\sigma_{x}\right\rangle
$$

where $\pi_{11}$ is the longitudinal piezoresistive coefficient for the crystallographic direction $\langle 100\rangle$ and $\left\langle\sigma_{x}\right\rangle$ is the mean stress in the resistor.

Now, considering that for a $1 / 4$ active Wheatstone bridge the relative variation of the output voltage $\left(\Delta \mathrm{V} / \mathrm{V}_{0}\right)$ corresponds to $1 / 4$ of $\Delta R / R_{0}$, the force sensitivity is

$$
\begin{aligned}
\mathfrak{R}_{F} & =\frac{\Delta V}{F} \\
& =\frac{1}{4} \frac{\Delta R}{R_{0}} \frac{\mathrm{V}_{\text {bias }}}{F} \\
& =-\frac{1}{16} \frac{E_{100 \pi_{11}}\left(2 L_{2}-L_{1}\right)\left(t_{R}+2 z_{0}\right)}{E I_{1}} V_{\text {bias }},
\end{aligned}
$$

where $E_{100}$ is the Young modulus of silicon along the $\langle 100\rangle$ direction, $t_{\mathrm{R}}$ is the thickness of the resistor (in this case we have approximated the profile of arsenic with a step profile after implantation and thermal treatment) and $\mathrm{V}_{\text {bias }}$ is the bias voltage applied to the bridge.

\section{Noise and MDF}

The MDF of the piezoresistive cantilevers is affected by two types of noise sources: electrical and thermomechanical. The two main electrical noises are thermal (or Johnson) noise and $1 / \mathrm{f}$ noise. The thermal noise has constant power density $\left(\mathrm{S}_{\mathrm{J}}\right)\left[\mathrm{V}^{2} / \mathrm{Hz}\right]$ for every frequency and is caused by the random collisions of the charge carriers. ${ }^{22}$ The $1 / \mathrm{f}$ noise is predominant at low frequency, the power density $\left(\mathrm{S}_{\mathrm{H}}\right)$ decreases as the inverse of the frequency and it is due to carriers mobility fluctuations. $^{23}$

On the other hand, the thermomechanical noise is due to the mechanical vibration of the cantilever caused by the brownian movement of the particles in and around the beam ${ }^{24,25}$ and in this case, the power density $\left(\mathrm{S}_{\mathrm{TH}}\right)$ for low quality factors and low frequencies is independent of the frequency. For piezoresistive microcantilevers, it is usually much smaller than the other two noise sources.

The total power noise density can be estimated by adding the three noise power densities,

$$
\mathrm{S}_{\mathrm{V}}=\mathrm{S}_{\mathrm{J}}+\mathrm{S}_{\mathrm{H}}+\mathrm{S}_{\mathrm{TH}}=4 k_{B} T R+\frac{\alpha \mathrm{V}_{\text {bias }}^{2}}{\mathrm{~N} f}+\mathfrak{R}_{F}^{2} \frac{4 k k_{B} T}{\omega_{0} Q},
$$

where $k_{B}$ is the Bolzmann constant, $T$ is the temperature, $R$ is the resistance value, $N$ is the number of carriers in the resistor, $f$ is the frequency, $\alpha$ is the Hooge coefficient, $\omega_{0}$ is the resonant frequency, and $\mathrm{Q}$ is the quality factor.

Integrating the total power density in a certain bandwidth, we can estimate the value of the noise voltage $\left(\sqrt{\left\langle\mathrm{V}^{2}\right\rangle}\right)$ and finally the minimum force that can be detected by the sensor
(MDF),

$$
\mathrm{MDF}=\frac{\sqrt{\left\langle\mathrm{V}^{2}\right\rangle}}{\mathfrak{R}_{F}^{2}}
$$

\section{CHARACTERIZATION METHODS}

Performing fast, accurate, and repeatable mechanical, electrical, and electromechanical characterization of the device properties is necessary in order to prove the reliability of the sensor and to validate the fabrication technology in view of future commercial applications. For these reasons, we decided to develop a setup that allows on-wafer properties characterization, which results in a considerable reduction of the testing time.

\section{A. Mechanical properties}

The spring constant of the cantilever is calculated according to Eq. (3). The thicknesses are measured using an optical interferometer (Nanospec AFT 200 from Nanometrics). The widths and lengths are verified using optical microscope and SEM images. The Young's modulus for silicon along $\langle 100\rangle$ direction is taken as $E_{S i}=130 \mathrm{GPa}^{26}$ and $E_{\mathrm{SiO} 2}=75 \mathrm{GPa}$ for thermal oxide. ${ }^{27}$ PECVD silicon nitride has been reported to present a wide range of values for its Young's modulus, from 60 to $300 \mathrm{GPa}^{28-30}$ depending on the deposition conditions and the layer thickness. Thus, it is necessary to experimentally determine such value for our material. We performed micromechanical measurements with a beam bending based technique ${ }^{31,32}$ on stiffer (specifically designed) test cantilevers (T1 design, see Table I) using a commercial AFM (Bruker Dimension 3100). The use of a profilometer ${ }^{33}$ is not possible for our cantilevers, due to their shape and their reduced dimensions of the cantilevers. From these measurements, it is easy to calculate the spring constants of the test cantilevers. Then, it is possible to extract the Young's modulus of the silicon nitride layer using Eqs. (3)-(5). When the AFM probe is in contact with a cantilever, in the contact point we can write the following equation to balance the forces (Fig. 3):

$$
F=k_{C} \delta_{C}=k_{P e f f} \delta_{P}
$$

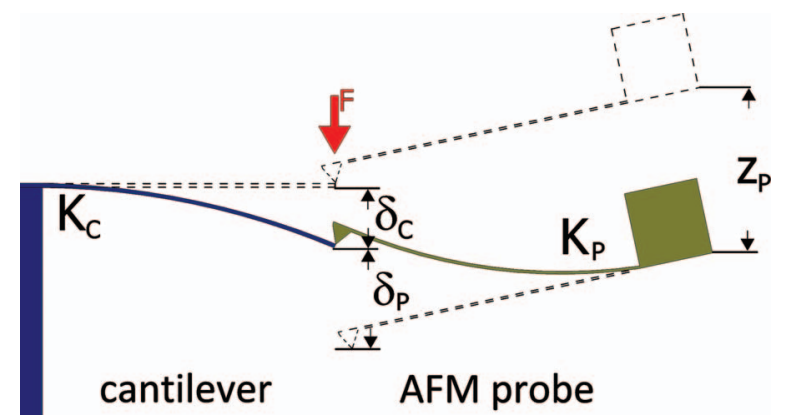

FIG. 3. (Color online) Beam bending spring constant measurement: the AFM probe (right) is deflecting the test cantilever (left). The initial cantilever and AFM probe positions are shown in dashed lines. 
TABLE I. Piezoresistive cantilevers: cantilever arms $\left(\mathrm{w}_{1}\right)$, total cantilever $\left(\mathrm{w}_{2}\right)$, and resistance $\left(\mathrm{w}_{\mathrm{R}}\right)$ widths, spring constant $k$, resistance mean value, square resistance mean value, and its relative standard deviation $(\sigma)$ and yield for the three different cantilever designs. Resistances and deviations are for substrate resistors and between brackets for cantilever resistors. T1 cantilevers are used for beam bending measurement.

\begin{tabular}{|c|c|c|c|c|c|c|c|c|c|}
\hline Design & $\begin{array}{l}\text { Number } \\
\text { of chips }\end{array}$ & $\begin{array}{c}\mathrm{w}_{1} \\
(\mu \mathrm{m})\end{array}$ & $\begin{array}{c}\mathrm{w}_{2} \\
(\mu \mathrm{m})\end{array}$ & $\begin{array}{l}\mathrm{w}_{\mathrm{R}} \\
(\mu \mathrm{m})\end{array}$ & $\begin{array}{c}k \\
(\mathrm{mN} / \mathrm{m})\end{array}$ & $\begin{array}{c}\text { Mean R } \\
(k \Omega)\end{array}$ & $\begin{array}{c}\text { Mean R } \\
(\Omega / s q)\end{array}$ & $\sigma$ & Yield \\
\hline B1 & 24 & 2 & 8 & 2 & 0.62 & $\begin{array}{c}16.3 \\
(18.3)\end{array}$ & $\begin{array}{l}100 \\
(113)\end{array}$ & $\begin{array}{l}3 \% \\
(6 \%)\end{array}$ & $100 \%$ \\
\hline B2 & 24 & 8 & 20 & 2 & 2.02 & $\begin{array}{c}16.7 \\
(18.1)\end{array}$ & $\begin{array}{c}99 \\
(107)\end{array}$ & $\begin{array}{c}3 \% \\
(6 \%)\end{array}$ & $100 \%$ \\
\hline B3 & 24 & 6 & 16 & 6 & 1.73 & $\begin{array}{c}6.8 \\
(7.3)\end{array}$ & $\begin{array}{c}126 \\
(134)\end{array}$ & $\begin{array}{l}3 \% \\
(6 \%)\end{array}$ & $100 \%$ \\
\hline $\mathrm{T} 1$ & 9 & 10 & 24 & & 24.0 & & & & \\
\hline
\end{tabular}

where $k_{C}$ and $\delta_{C}$ are the spring constant and deflection of the cantilever while $k_{P e f f}$ and $\delta_{P}$ are the effective spring constant $\left(k_{\text {Peff }}=k_{P} / \cos ^{2} \alpha\right.$ and $\alpha=12^{\circ}$ is the mounting angle of the probe) and the deflection of the AFM probe. We know also that the total probe displacement $\left(z_{P}\right)$ is the sum of the deflection of the probe and the one of the cantilever under test,

$$
z_{P}=\delta_{C}+\delta_{P},
$$

therefore we can write

$$
k_{C}=k_{P e f f} \frac{\delta_{P}}{\delta_{C}}=k_{P e f f} \frac{\delta_{P}}{z_{P}-\delta_{P}}=k_{P e f f} \frac{\tan \theta}{1-\tan \theta},
$$

where $\tan \theta$ is the slope of the deflection curve of the AFM probe while deflecting the cantilever (Fig. 4).

For these measurements we used an AFM probe with a measured spring constant $K_{\text {Peff }}=(0.055 \pm 0.002) \mathrm{N} / \mathrm{m}$ (calibrated using Sader's tune method ${ }^{34}$ ) in agreement with the theoretical one $(0.05 \mathrm{~N} / \mathrm{m})$. We initially performed multiple force curves on a single cantilever to study the repeatability of the method from which we obtained a relative standard

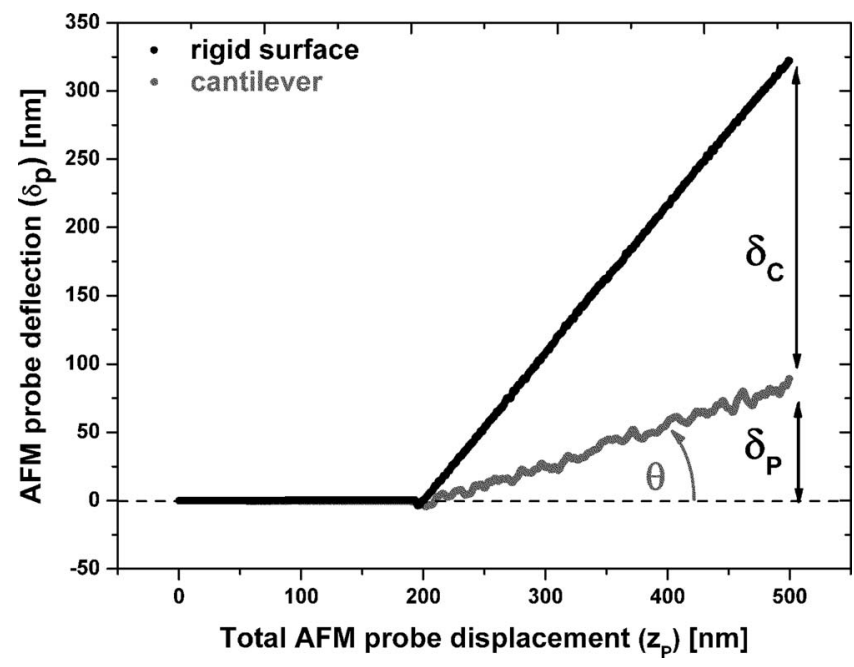

FIG. 4. AFM probe deflection curves $\left(\delta_{\mathrm{P}}\right)$ against the total AFM probe displacement $\left(z_{\mathrm{C}}\right)$. In black the AFM probe is deflected against a stiff surface. This curve is used to calibrate the AFM photodetector sensitivity. In gray the AFM probe is deflected against the cantilever under test: the difference between the two curves represent the deflection of the cantilever under test $\left(\delta_{\mathrm{C}}\right)$. deviation of $5 \%$. This was then repeated on 9 cantilevers, to study the variation of the spring constant in different points of the wafer. The mean value of the slope, for the different cantilevers, is $\tan \theta=0.306$ (with a relative standard deviation of 6\%) that indicates a spring constant $k_{C}=(0.0240$ $\pm 0.0014) \mathrm{N} / \mathrm{m}$ and therefore an $E_{n i t}=(70 \pm 7)$ Gpa. This value was confirmed measuring the Young's modulus of the $100 \mathrm{~nm}$ thick silicon nitride layer on silicon oxide and aluminum substrate by the nanoindentantion technique (Nano Indenter XP MTS). With this value of the silicon nitride Young modulus, we calculated the spring constant for the different cantilever designs. Note that the rest of piezoresistive cantilever designs have much lower spring constants and it was therefore not possible to perform the spring constant calibration directly on them with this method. The key dimensions and $k$ values of the cantilevers are depicted in the caption of Fig. 1 and in Table I.

\section{B. Electrical properties}

The electrical characteristics of the piezoresistors are measured using a semiautomatic probe station (Karl Süss PA200) and a semiconductor parameter analyzer HP4155. I-V curves are acquired for every tested resistance by applying a bias voltage ranging between $-5 \mathrm{~V}$ and $5 \mathrm{~V}$ while recording the current. We find a good ohmic contact between aluminum and n-type silicon, very high fabrication yield ( $>95 \%)$, and low relative standard deviation of the resistance values for both the resistors implanted in the substrate $(3 \%)$ and the ones integrated on the cantilever (6\%) (Table I). The statistics are made out of 24 chips per each design.

The noise power spectral density is measured by using a low noise amplifier (LNA) (SR560 - Stanford Research) and a dynamic analyzer (SR785 - Stanford Research). The pads are contacted by using the probe card specifically designed for the sensitivity characterization (see next paragraph). The measurements are performed in the same probe station used before, which provides also a good electronic shielding from external noise sources. The wiring system and the supply voltage are adapted to reduce as much as possible the nonintrinsic noise. ${ }^{35}$ For this purpose, we use 4 rechargeable batteries (Eneloop - Sanyo) that give a bias voltage $\mathrm{V}_{\mathrm{CC}}=5 \mathrm{~V}$ and as short as possible coaxial cables. The differential signal is amplified 1000 times by the LNA and the spectra recorded 


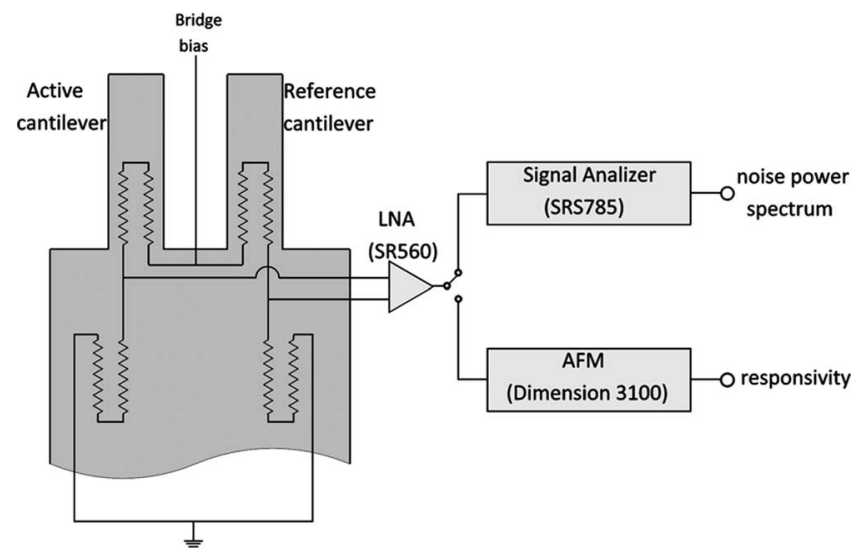

FIG. 5. Noise and sensitivity measurements electrical scheme. The differential voltage of the Wheatstone bridge is amplified by the low noise voltage amplifier. The noise spectrum is recorded by the signal analyzer while the sensitivity signal is recorded by the AFM electronics.

by the dynamic analyzer (Figs. 5 and 6). First we tested the repeatability of the measurement, recording the noise spectrum 9 times for 3 different chips obtaining a maximum relative standard deviation of $2 \%$ and then we measured the noise spectra of all the chips across the wafer. In Table II, we report the mean voltage noise value referred to input $\left(\mathrm{V}_{\text {noise }}\right)$ measured between $1 \mathrm{~Hz}$ and $1 \mathrm{kHz}$.

\section{Sensitivity and MDF}

In order to determine the device sensitivity and therefore the MDF in a fast way, we developed a specific setup to measure it directly on wafer level. For this purpose, we designed and fabricated a special probe-card (Fig. 7) that allows us to contact the pads of our chips and simultaneously deflect them by other means. In particular, its reduced size permits the use of an AFM (dimension 3100) to deflect our cantilevers, thus

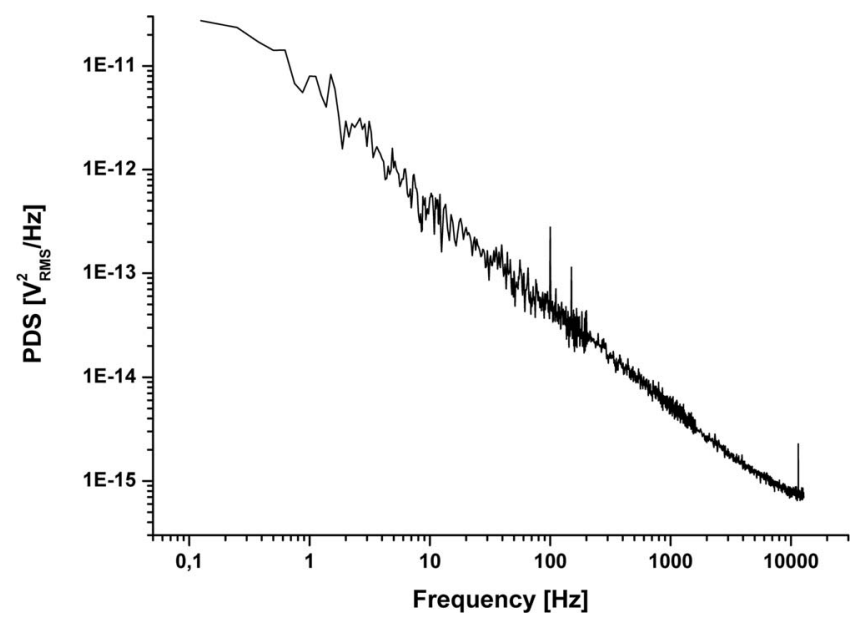

FIG. 6. Input referred noise power spectral density for the Wheatstone bridge (design B2). Integrating the noise between $1 \mathrm{~Hz}$ and $1 \mathrm{kHz}$, we obtain a noise average value of $5.94 \mu \mathrm{V}$. The bridge is $5 \mathrm{~V} \mathrm{DC}$ biased and the pads are contacted by the probe card. Values of the resistances are reported in Table I.
TABLE II. Piezoresistive cantilever: deflection sensitivity $(\Delta \mathrm{V} / \delta)$ at $140 \mu \mathrm{m}$ from the clamping edge, gauge factor $(\mathrm{G})$, mean force sensitivity $\left(\Re_{F}\right)$ for a force applied at the tip and relative standard deviation $(\sigma)$, mean voltage noise value referred to input $\left(\mathrm{V}_{\text {noise }}\right)$ measured between $1 \mathrm{~Hz}$ and $1 \mathrm{kHz}$ and minimum detectable force (MDF). Statistics were made on 24 chips per each design.

\begin{tabular}{lcccccc}
\hline Design & $\begin{array}{c}\Delta \mathrm{V} / \delta \\
(\mu \mathrm{V} / \mu \mathrm{m})\end{array}$ & $\mathrm{G}$ & $\begin{array}{c}\mathfrak{R}_{F} \\
(\mu \mathrm{V} / n \mathrm{~N})\end{array}$ & $\sigma$ & $\begin{array}{c}\mathrm{V}_{\text {noise }} 1 \mathrm{~Hz}-1 \mathrm{kHz} \\
(\mu \mathrm{V})\end{array}$ & $\begin{array}{c}\mathrm{MDF} \\
(p \mathrm{~N})\end{array}$ \\
\hline $\mathrm{B} 1$ & 241 & 102 & 158 & $8 \%$ & 5.87 & 37 \\
$\mathrm{~B} 2$ & 231 & 102 & 46 & $8 \%$ & 5.94 & 129 \\
$\mathrm{~B} 3$ & 230 & 102 & 53 & $8 \%$ & 4.95 & 93 \\
\hline \hline
\end{tabular}

benefiting from the high motional precision of such equipment.

In our setup, we use the batteries and the LNA SR 560 for biasing the bridge and amplifying the output signal, respectively. We place the whole wafer on the chuck of the AFM. In Fig. 8, it is possible to see how the AFM probe can contact the to-be-tested cantilevers. A stiff AFM probe $(k \approx 40 \mathrm{~N} / \mathrm{m})$ is used to deflect the cantilevers at a certain distance from the clamping edge (namely at $140 \pm 3 \mu \mathrm{m}$ or at the free end). The output differential voltage is amplified and recorded continuously together with the AFM probe displacement (Fig. 9). In order to precisely position the AFM tip on top of the device and work under low interaction forces, the AFM is operated in dynamic mode. In this mode, the tipsample interaction is controlled by maintaining a fixed amplitude of the oscillation of the AFM cantilever at its resonance frequency. This gives us another advantage and is the ability to detect when the AFM probe contacts the piezoresistive cantilever, which is when the oscillation amplitude drops to zero. Since the stiffness of the AFM probe is much bigger than the one of the cantilever under investigation (4 or 5 orders of magnitude), its static deflection is negligible. After one measurement that typically is performed at $1 \mathrm{~Hz}$, the probe card can be moved to the next chip under investigation by using a manual micromanipulator mounted onto the AFM chuck.

The probe-card presented here is specifically designed to be used in the dimension 3100 AFM, can access all the

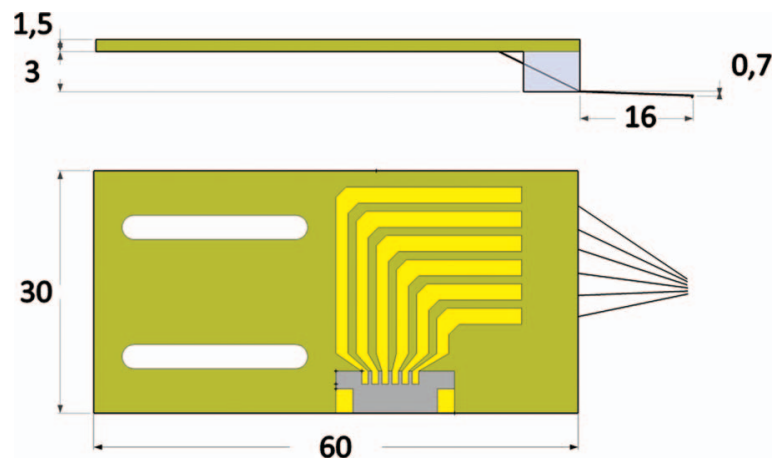

FIG. 7. (Color online) Lateral view (up) and top view (down) of the probe card design (dimensions in $\mathrm{mm}$ ). For the probes we fixed the maximum vertical drop to $0.7 \mathrm{~mm}$ and the length to $16 \mathrm{~mm}$ to avoid any interaction with the AFM head. 


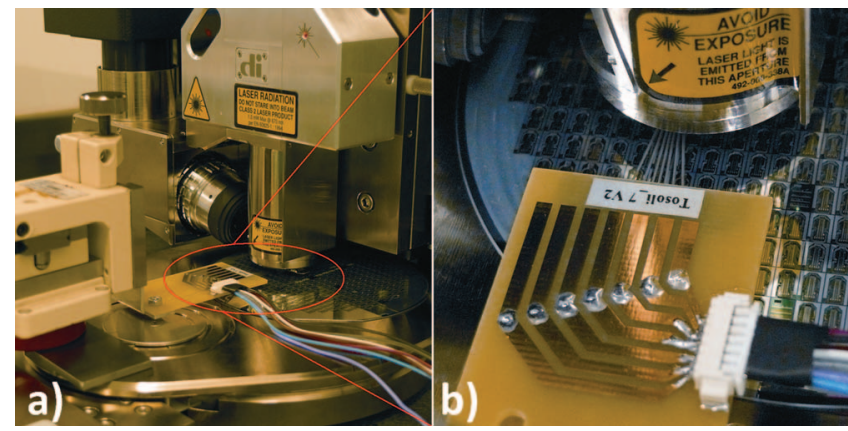

FIG. 8. (Color online) (a) The probe card is held by a micromanipulator onto the chuck of the AFM. (b) The probes are contacting the pads of the chip while the AFM tip performs the displacement of the cantilever for measuring the sensitivity.

devices across the 4" wafer and the probes are correctly aligned onto the chip pads using the optical microscope of the AFM. Anyway it can be used (or adapted to) in many other equipments, as for example the probe station KS PA200 (that we already demonstrated in the Sec. III B). The probes are long and almost horizontal in order to avoid any interaction with the AFM head. In addition, the PCB (printed circuit board) has to be small to fit onto the AFM chuck. The two grooves (visible in the left part of the top view) increase the travel range of the micropositioner. The use of the probe-card together with a manual micromanipulator allows characterizing a considerable number of chips (24 per each design) in a relatively short time (10 chip/h) across the whole wafer. This constitutes a definite improvement with the way these measurements were done up to date, ${ }^{19}$ where the chip had to be pulled apart from the wafer, attached on a PCB board and wire bonded, which is a time consuming, risky and not robust processes. In addition, with the new method we reduced also the risk to damage the sensor. An additional way to decrease the measurement time would be to automate in part the measurement setup using a motorized micromanipulator. However, there is a limit to the amount of the automation due to the complex nature of the measurement.

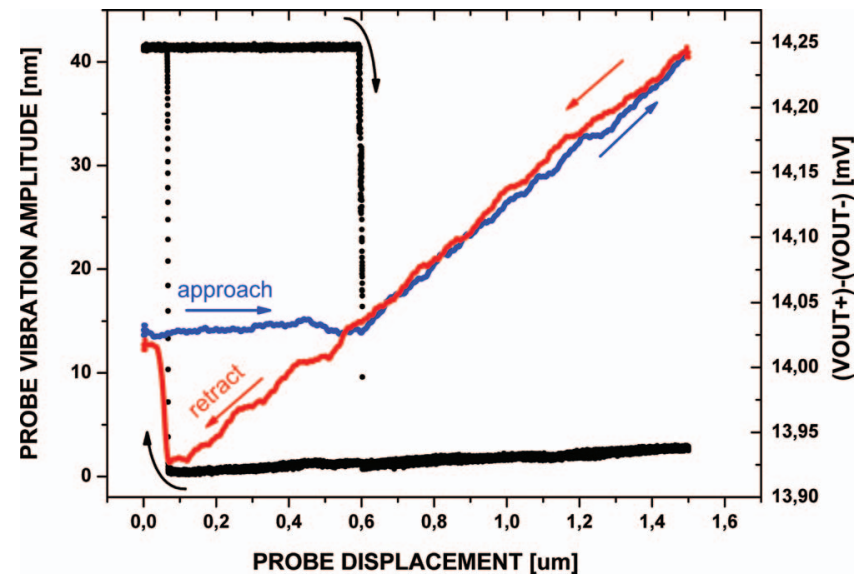

FIG. 9. (Color online) Electromechanical response of a piezoresistive cantilever (design B2) with no amplification. At a length of $140 \mu \mathrm{m}$, a stiff probe (in tapping mode) starts to deflect downwards the cantilever when the vibration decreases from around $40 \mathrm{~nm}$ to almost $0 \mathrm{~nm}$. At the same time the output differential voltage of the Wheatstone bridge (WB) starts to increase (approach line) from $14.03 \mathrm{mV}$ to $14.24 \mathrm{mV}$ for a deflection of $0.91 \mu \mathrm{m}$. This means a displacement sensitivity of around $231 \mu \mathrm{V} / \mathrm{um}$ at the deflection point and a force sensitivity of $46 \mu \mathrm{V} / n \mathrm{~N}$ at the tip. Considering the noise value of $5.94 \mu \mathrm{V}$, we can assure a MDF $=129 p \mathrm{~N}$. In the retract curve is also visible the strong interaction between the AFM tip and the cantilever under test which is bending upwards $0.5 \mu \mathrm{m}$. The force curve (approach and retract) is performed over $1 \mathrm{~s}$.

Figure 9 shows a typical deflection experiment. From these measurements, we obtain the displacement sensitivity $(\Delta \mathrm{V} / \delta)$ and we can calculate the gauge factor $(\mathrm{G})$ and the force sensitivity $\left(\mathfrak{R}_{F}=\Delta \mathrm{V} / \mathrm{F}\right)$ for a force $\mathrm{F}$ applied in the end of the cantilever (Eq. (7)). This technique allows a precise positioning of the tip along the cantilever thanks to the optical microscope and to the motorized positioning stage with a repeatability of $\pm 3 \mu \mathrm{m}$, and a very good control of the deflection, due to the piezoactuator of the AFM. In order to check the repeatability of the measurement method, we performed multiple force curves on different piezoresistive cantilevers and we obtained a maximum relative standard deviation of $5 \%$ for the displacement sensitivity. This variability is mainly
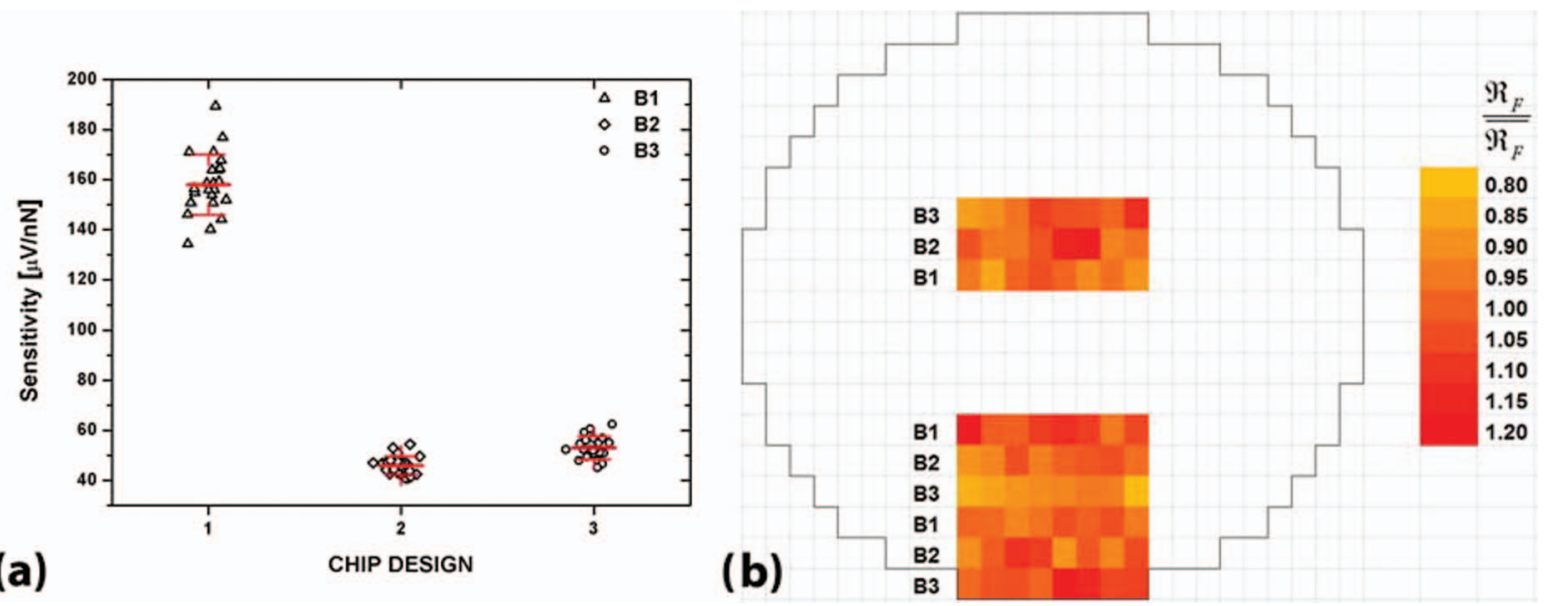

FIG. 10. (Color online) (a) Sensitivity results for 72 chips (24 per each design). Triangles, squares, and circles refer respectively to the designs B1, B2, and B3. Averaged values and standard deviation are reported in red for each design. (b) Sensitivity wafer-map. The 24 chips of each design are positioned in 3 different rows of 8 chips each. The relative sensitivity (sensitivity divided by the averaged sensitivity, $\Re_{F} / \overline{\Re_{F}}$ ) increases as reported in the legend. 
due to two sources: the low frequency noise of the piezoresistors and the uncertainty of the positioning system. In addition to the repeatability, we studied also the variation of the sensitivity of the chips across the wafer (Fig. 10) and in Table II, we report the mean value of the sensitivities, noise and MDF. This shows that our measurement system is particularly suited to technology characterization where several device types and several device geometries within a type have to be measured. For the best design we calculate a minimum detectable force of $37 p N$. The high sensitivity, MDF, yield, and the relatively small deviation in the characteristics are very promising and show a reliable process technology.

\section{CONCLUSIONS}

We have presented high throughput characterization methodologies and setups for the on-wafer measurement of mechanical, electrical, and electromechanical characteristics of MEMS piezoresistive cantilever force sensors. The setup is based on the use of atomic force microscope technology (providing high displacement precision) and on a specific probe card that can be integrated within the AFM chuck to drastically reduce the testing time. The development of a fast and reliable on-wafer complete characterization method of the cantilevers was necessary to validate the process technology in view of possible applications. For the best design, the force sensitivity, noise, and MDF are $158 \mu \mathrm{V} / n \mathrm{~N}, 5.81 \mu \mathrm{V}$ $(1 \mathrm{~Hz}-1 \mathrm{kHz})$, and $37 p \mathrm{~N}$, respectively and the device parameter relative standard deviation is $8 \%$. These characteristics allow the cantilevers to be applied as biomolecular detectors for the measurement of the intermolecular binding forces between ligands and receptors that are in the order of hundreds of piconewton for a single pair. ${ }^{36,37}$

\section{ACKNOWLEDGMENTS}

The authors would like to thank Jordi Sacristán Riquelme and Carlos José Camargo Barbosa for the useful discussions during the development of the setup for the noise measurements and for the beam bending technique, respectively. This work has been supported by MICINN through projects TEC2011-23600 and NANOSELECT-CSD200700041 (Consolider-Ingenio 2010 Programme).

${ }^{1}$ N. V. Lavrik, M. J. Sepaniak, and P. G. Datskos, Rev. Sci. Instrum. 75, 2229 (2004).

${ }^{2}$ K. C. Neuman and A. Nagy, Nat. Methods 5, 491 (2008).

${ }^{3}$ A. A. Barlian, W. T. Park, J. R. Mallon, A. J. Rastegar, and B. L. Pruitt, Proc. IEEE 97, 513 (2009).

${ }^{4}$ M. Tortonese, R. C. Barrett, and C. F. Quate, Appl. Phys. Lett. 62, 834 (1993).
${ }^{5}$ R. Linnemann, T. Gotszalk, I. W. Rangelow, P. Dumania, and E. Oesterschulze, J. Vac. Sci. Technol. B 14, 856 (1996).

${ }^{6}$ Y. Su, A. G. R. Evans, A. Brunnschweiler, G. Ensell, and M. Koch, Sens. Actuators, A 60, 163 (1997).

${ }^{7}$ R. Jumpertz, A. v. d. Hart, O. Ohlsson, F. Saurenbach, and J. Schelten, Microelectron. Eng. 41-42, 441 (1998).

${ }^{8}$ J. Thaysen, A. Boisen, O. Hansen, and S. Bouwstra, Sens. Actuators, A 83, 47 (2000); J. Thaysen, Ph.D. dissertation, Technical University of Denemark, 2001.

${ }^{9}$ M. Willemin, C. Rossel, J. Brugger, M. H. Despont, H. Rothuizen, P. Vettiger, J. Hofer, and H. Keller, J. Appl. Phys. 83, 1163 (1998).

${ }^{10}$ J. A. Harley and T. W. Kenny, Appl. Phys. Lett. 75, 289 (1999); J. A. Harley, Ph.D. dissertation, Stanford University, 2000.

${ }^{11}$ B. L. Pruitt and T. W. Kenny, Sens. Actuators, A 104, 68 (2003).

${ }^{12}$ J. L. Arlett, J. R. Maloney, B. Gudlewski, M. Muluneh, and M. L. Roukes, Nano Lett. 6, 1000 (2006); J. Arlett, Ph.D. dissertation, California Institute of Technology, 2006.

${ }^{13}$ G. Villanueva, F. Perez-Murano, M. Zimmermann, J. Lichtenberg, and J. Bausells, Microelectron. Eng. 83, 1302 (2006).

${ }^{14}$ G. Villanueva, J. A. Plaza, J. Montserrat, F. Perez-Murano, and J. Bausells, Microelectron. Eng. 85, 1120 (2008).

${ }^{15}$ G. Tosolini, G. Villanueva, F. Perez-Murano, and J. Bausells, Microelectron. Eng. 87, 1245 (2010).

${ }^{16}$ Y. Kanda, Sens. Actuators, A 28, 83 (1991).

${ }^{17}$ T. Akiyama, A. Tonin, H.-R. Hidber, J. Brugger, P. Vettiger, U. Staufer, and N. F. de Rooij, Sens. Actuators, A 64, 1 (1998).

${ }^{18}$ R. Linnemann, T. Gotszalk, L. Hadjiiski, and I. W. Rangelow, Thin Solid Films 264, 159 (1995).

${ }^{19}$ G. Villanueva, J. Montserrat, F. Perez-Murano, G. Rius, and J. Bausells, Microelectron. Eng. 73-74, 480 (2004).

${ }^{20}$ S.-J. Park, J. C. Doll, and B. L. Pruitt, J. Microelectromech. Syst. 19, 137 (2010).

${ }^{21}$ S.-J. Park, J. C. Doll, A. J. Rastegar, and B. L. Pruitt, J. Microelectromech. Syst. 19, 149 (2010).

${ }^{22}$ H. Nyquist, Phys. Rev. 32, 110 (1928).

${ }^{23}$ F. N. Hooge, IEEE Trans. Electron Devices 41, 1926 (1994).

${ }^{24}$ P. R. Saulson, Phys. Rev. D 42, 2437 (1990).

${ }^{25}$ J. A. Harley and T. W. Kenny, J. Microelectromech. Syst. 9, 226 (2000).

${ }^{26}$ J. J. Wortman and R. A. Evans, J. Appl. Phys. 36, 153 (1965).

${ }^{27}$ B. Halg, Proceeding of IEEE Micro Electro Mechanical Systems, Napa Valley, California, USA, 1990 (Landis \& Gyr Betriebs AG, Zug, Switzerland, 1990), p. 172.

${ }^{28}$ K. B. Gavan, H. J. R. Westra, E. van der Drift, W. J. Venstra, and H. S. J. van der Zant, Appl. Phys. Lett. 94, 233108 (2009).

${ }^{29}$ H. Huang, K. J. Winchester, A. Suvorova, B. R. Lawn, Y. Liu, X. Z. Hu, J. M. Dell, and L. Faraone, Mater. Sci. Eng., A 435-436, 453 (2006).

${ }^{30}$ G. J. McShane, M. Boutchich, A. S. Phani, D. F. Moore, and T. J. Lu, J. Micromech. Microeng. 16, 1926 (2006).

${ }^{31}$ H. Campanella, CJ. Camargo, J. Montserrat, and J. Esteve, Proceeding of 21st Micromechanics and Micro systems Europe Workshop, Enschede, Netherland, 2010, edited by L. Abelmann, H. Groenland, J. van Honschoten, and H. Veputten (University of Twente, Enschede, The Netherlands, 2010), p. 181.

${ }^{32}$ E. D. Langlois, G. A. Shaw, J. A. Kramar, J. R. Pratt, and D. C. Hurley, Rev. Sci. Instrum. 78, 093705 (2007).

${ }^{33}$ M. Hopcroft, T. Kramer, G. Kim, K. Takashima, Y. Higo, D. Moore, and J. Brugger, Fatigue Fract. Eng. Mater. Struct. 28, 735 (2005).

${ }^{34}$ J. E. Sader, J. Appl. Phys. 84, 64 (1998).

${ }^{35}$ R. Dieme, J. Acoust. Soc. Am. 119, 2710 (2006); R. Dieme, M.S. dissertation, University of Florida, Gainesville, 2005.

${ }^{36} \mathrm{P}$. Hinterdorfer, W. Baumgartner, H. J. Gruber, K. Schilcher, and H. Schindler, Proc. Natl. Acad. Sci. U.S.A. 93, 3477 (1996).

${ }^{37}$ C.-K. Lee, Y.-M. Wang, L.-S. Huang, and S. Lin, Micron 38, 446 (2007). 